\title{
Magnesium supplementation improves diabetic mitochondrial and cardiac diastolic function
}

\author{
Man Liu, ${ }^{1}$ Euy-Myoung Jeong, ${ }^{2,3}$ Hong Liu, ${ }^{1}$ An Xie, ${ }^{1}$ Eui Young So, ${ }^{2,3}$ Guangbin Shi, ${ }^{2}$ Go Eun Jeong, ${ }^{4}$ \\ Anyu Zhou, ${ }^{2,3}$ and Samuel C. Dudley Jr. ${ }^{1}$ \\ 'Division of Cardiology, Department of Medicine, the Lillehei Heart Institute, University of Minnesota at Twin Cities, \\ Minneapolis, Minnesota. ${ }^{2}$ Cardiovascular Research Center, Lifespan Rhode Island Hospital, Providence, Rhode Island. ${ }^{3}$ The \\ Warren Alpert Medical School, Brown University, Providence, Rhode Island. ${ }^{4}$ Brown University, Providence, Rhode Island.
}

\begin{abstract}
In heart failure and type $\mathbf{2}$ diabetes mellitus (DM), the majority of patients have hypomagnesemia, and magnesium $(\mathrm{Mg})$ supplementation has improved cardiac function and insulin resistance. Recently, we have shown that DM can cause cardiac diastolic dysfunction (DD). Therefore, we hypothesized that $\mathrm{Mg}$ supplementation would improve diastolic function in DM. High-fat dietinduced diabetic mouse hearts showed increased cardiac DD and hypertrophy. Mice with DM showed a significantly increased $\mathrm{E} / \mathrm{e}$ ' ratio (the ratio of transmitral Doppler early filling velocity [E] to tissue Doppler early diastolic mitral annular velocity [e']) in the echocardiogram, left ventricular end diastolic volume (LVEDV), incidence of DD, left ventricular posterior wall thickness in diastole (PWTd), and ratio of heart weight to tibia length (HW/TL) when compared with controls. DM mice also had hypomagnesemia. Ventricular cardiomyocytes isolated from DM mice exhibited decreased mitochondrial ATP production, a 1.7- \pm 0.2-fold increase of mitochondrial ROS, depolarization of the mitochondrial membrane potential, and mitochondrial $\mathrm{Ca}^{2+}$ overload. Dietary $\mathrm{Mg}$ administration (50 $\mathrm{mg} / \mathrm{ml}$ in the drinking water) for 6 weeks increased plasma Mg concentration and improved cardiac function. At the cellular level, Mg improved mitochondrial function with increased ATP, decreased mitochondrial ROS and $\mathrm{Ca}^{2+}$ overload, and repolarized mitochondrial membrane potential. In conclusion, Mg supplementation improved mitochondrial function, reduced oxidative stress, and prevented DD in DM.
\end{abstract}

Conflict of interest: The authors have declared that no conflict of interest exists.

License: Copyright 2019, American Society for Clinical Investigation.

Submitted: June 26, 2018

Accepted: December 5, 2018

Published: January 10, 2019

Reference information:

JCI Insight. 2019;4(1):e123182. https:// doi.org/10.1172/jci.insight.123182.

\section{Introduction}

A high-fat diet (HFD) and a sedentary lifestyle have been implicated in the global epidemic of obesity $(1,2)$. Obesity and diabetes mellitus (DM) are important risk factors for the development of cardiovascular disease (1, 3), including cardiac diastolic dysfunction (DD) (4-7). Cardiac DD is thought to be one pathological mechanism underlying heart failure with preserved ejection fraction. Altered myocardial metabolism and increased oxidative stress accompany DM-associated DD (3, 7-10). Recently, we have reported that cardiac mitochondrial oxidative stress can cause DD and heart failure with preserved ejection fraction by oxidatively modifying cardiac myosin binding protein $C(10-13)$.

Magnesium $(\mathrm{Mg})$ is an essential element for mitochondrial function, especially for ATP production $\mathrm{Mg}$ deficiency is found commonly in cardiovascular disease, type $2 \mathrm{DM}$, hypertension, heart failure, and ventricular arrhythmia patients (14-17). A low serum $\mathrm{Mg}$ level is associated with increased cardiovascular mortality (15), while increased dietary $\mathrm{Mg}$ intake is associated with a decreased risk of developing type 2 $\mathrm{DM}$ and heart failure $(8,18,19)$. We investigated whether oral $\mathrm{Mg}$ supplementation would improve mitochondrial dysfunction and DD in a HFD mouse model of type 2 DM.

\section{Results}

HFD induces DM in mice. As we have shown previously (20), HFD induced DM, and mice on this diet showed significant obesity (Table 1$)$. The body weight/tibia length (BW/TL) ratio was increased significantly from $14.5 \pm 0.4 \mathrm{~g} / \mathrm{cm}$ of control mice $(\mathrm{CT})$ to $19.3 \pm 0.4 \mathrm{~g} / \mathrm{cm}$ of HFD-fed mice $(P<0.0001)$. HFDfed mice showed elevated fasting serum insulin $(32.0 \pm 0.5 \mathrm{mU} / 1$ in $\mathrm{CT}$ vs. $50.6 \pm 5.7 \mathrm{mU} / \mathrm{L}$ in $\mathrm{HFD}$-fed 
Table 1. HFD induced DM and DD in mice and decreased plasma and cytosol Mg levels

\begin{tabular}{|c|c|c|c|c|c|c|c|c|}
\hline \multirow[b]{2}{*}{ DM indicators } & \multicolumn{2}{|c|}{ Ст } & \multicolumn{2}{|c|}{$\mathrm{CT}+\mathrm{Mg}$} & \multicolumn{2}{|c|}{ DM } & \multicolumn{2}{|c|}{$\mathrm{DM}+\mathrm{Mg}$} \\
\hline & Mean \pm SEM & $n$ & Mean \pm SEM & $n$ & Mean \pm SEM & $n$ & Mean \pm SEM & $n$ \\
\hline Body weight (g) & $30 \pm 1$ & 10 & $31 \pm 1$ & 10 & $45 \pm 1^{A}$ & 10 & $40 \pm 1^{A, B}$ & 10 \\
\hline Body weight/tibia length (g/cm) & $14.5 \pm 0.4$ & 10 & $14.6 \pm 0.4$ & 10 & $19.3 \pm 0.4^{A}$ & 10 & $17.8 \pm 0.5^{A, B}$ & 10 \\
\hline Fasting blood glucose (mg/dl) & $197 \pm 6$ & 5 & $201 \pm 6$ & 5 & $270 \pm 17^{c}$ & 5 & $216 \pm 7^{B}$ & 5 \\
\hline Fasting Serum Insulin (mU/l) & $32.0 \pm 0.5$ & 5 & $37.9 \pm 1.7$ & 5 & $50.6 \pm 5.7^{\circ}$ & 5 & $45.2 \pm 4.6^{D}$ & 5 \\
\hline Heart weight/tibia length (mg/cm) & $60 \pm 1$ & 10 & $59 \pm 1$ & 10 & $72 \pm 2^{A}$ & 10 & $64 \pm 1^{D, F}$ & 10 \\
\hline Heart weight (mg) & $124 \pm 2$ & 10 & $126 \pm 2$ & 10 & $168 \pm 4^{A}$ & 10 & $146 \pm 3^{\mathrm{C}, \mathrm{H}}$ & 10 \\
\hline LVEDV $(\mu \mathrm{l})$ & $59.3 \pm 2.6$ & 6 & $65.5 \pm 1.8$ & 7 & $78.9 \pm 1.0^{A}$ & 6 & $53.7 \pm 2.9^{H}$ & 10 \\
\hline PWTd (mm) & $0.77 \pm 0.03$ & 5 & $0.70 \pm 0.04$ & 7 & $0.93 \pm 0.02^{c}$ & 5 & $0.77 \pm 0.02^{\mathrm{E}}$ & 5 \\
\hline Plasma Mg (mmol/l) & $1.00 \pm 0.03$ & 18 & $1.61 \pm 0.06^{A}$ & 15 & $0.80 \pm 0.04^{\complement}$ & 12 & $1.49 \pm 0.05^{A, H}$ & 7 \\
\hline
\end{tabular}

Data are shown as mean \pm SEM, and $n$ is the animal number used in each group. One-way ANOVA with Bonferroni post hoc tests and 2-tailed Student's $t$ tests were used. CT, control mice; $C T+M g$, control mice fed with normal chow and $\mathrm{Mg}$ in drinking water; DM, diabetic mice fed with high-fat diet; DM+Mg, mice fed with high-fat diet and Mg in drinking water; E/e', the ratio of transmitral Doppler early filling velocity to tissue Doppler early diastolic mitral annular velocity; HFD, high-fat diet; LVEDV, left ventricular end diastolic volume; Mg, magnesium; PWTd, left ventricular diastolic posterior wall thickness. ${ }^{A} P<0.0001$ (vs. CT); ${ }^{\mathrm{B}} P<0.05$ (vs. DM); ${ }^{\mathrm{C}} P<0.01$ (vs. CT); ${ }^{\mathrm{D}} P<0.05$ (vs. CT); ${ }^{\mathrm{E}} P<0.001$ (vs. DM); ${ }^{\mathrm{F}} P<0.01$ (vs. DM); ${ }^{\mathrm{C}} P<0.001$ (vs. CT); ${ }^{\mathrm{H}} P<0.0001$ (vs. DM).

mice, $P<0.05)$ and fasting blood glucose levels $(197 \pm 6 \mathrm{mg} / \mathrm{dl}$ in CT vs. $270 \pm 17 \mathrm{mg} / \mathrm{dl}$ in HFD-fed mice, $P<0.001)$. Additionally, the glucose tolerance test following i.p. glucose administration showed significantly prolonged elevation of blood glucose in the HFD-fed mice compared with the CT ( $n=5$ for both groups, Figure 1).

$D M$ induced cardiac DD and hypertrophy. HFD-induced DM mice exhibited cardiac DD and hypertrophy, as shown in Table 1. The ratio of transmitral Doppler early filling velocity (E) to tissue Doppler early diastolic mitral annular velocity (e') (E/e') was significantly higher in DM mice. The ratio of heart weight/TL (HW/TL), the left ventricular end diastolic volume (LVEDV), and the left ventricular posterior wall thickness in diastole (PWTd) were significantly increased in DM mice compared with $\mathrm{CT}$. These data indicate cardiac DD and hypertrophy. The ejection fraction was preserved in DM mice $(60.0 \% \pm 5.2 \%$ vs. $63.7 \% \pm 4.2 \%$ of CT, $P=0.30)$. DM was also associated with decreased Mg concentration in the plasma $(80.0 \% \pm 4.7 \%$ of CT, $P<0.001)$ and in the cytosol $(87.2 \% \pm 0.0 \%$ of CT, $P$ $<0.0001$ ), as listed in Table 1 .

Dietary $\mathrm{Mg}$ supplementation increased plasma Mg concentration and improved DM. Low $\mathrm{Mg}$ has been associated with increased heart failure $(15,17)$, and DM mice had a low plasma Mg concentration. Therefore, $\mathrm{Mg}$ supplementation was given to DM mice $(\mathrm{DM}+\mathrm{Mg})$ in the drinking water $(50 \mathrm{mg} / \mathrm{ml})$ for an additional 6 weeks (21). As shown in Figure 2A and Table 1, dietary Mg raised the plasma Mg level in DM mice to $1.49 \pm 0.05 \mathrm{mmol} / 1(P<0.0001$ vs. $0.80 \pm 0.04 \mathrm{mmol} / 1$ in untreated DM mice). The $\mathrm{DM}+\mathrm{Mg}$ group had a plasma $\mathrm{Mg}$ concentration similar to $\mathrm{Mg}$-treated $\mathrm{CT}(\mathrm{CT}+\mathrm{Mg})(1.61 \pm 0.06 \mathrm{mmol} / \mathrm{l}$ in $\mathrm{CT}+\mathrm{Mg}$ mice,

Figure 1. HFD induced DM with elevated glucose levels as compared with controls during the i.p. glucose tolerance test. Five animals were tested for each group. The 2-tailed Student's $t$ test was used; ${ }^{*} P<0.01$ vs. control. CT, control mice; DM, diabetic mice fed with high-fat diet.

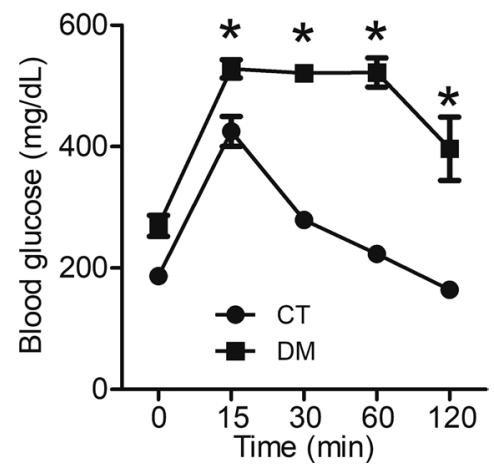



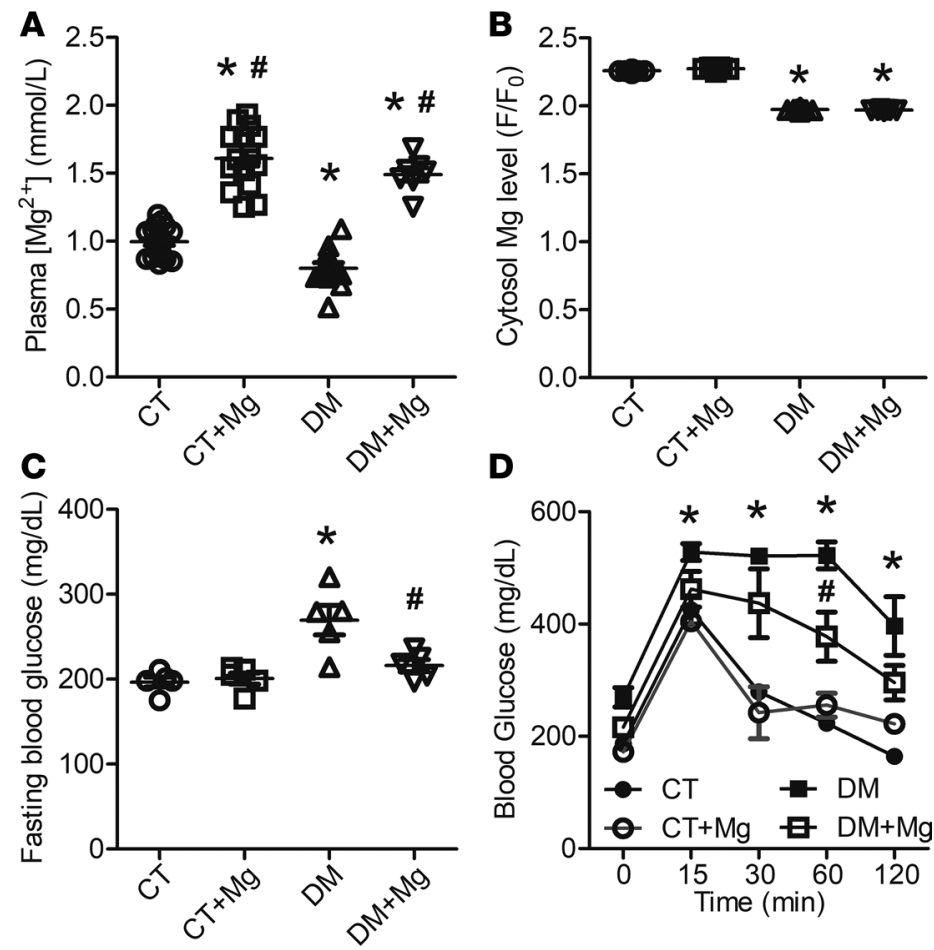

Figure 2. Effects of dietary Mg on DM mice. (A) plasma Mg concentration was decreased in DM mice and reinstated by dietary Mg. Seven to 15 animals were tested for each group. (B) The cytosol free Mg level was decreased in DM mice, and dietary Mg did not recover it. Cardiomyocytes isolated from the left ventricle of 7-15 animals were tested for each group. (C) Dietary Mg decreased the fasting blood glucose in DM mice. Five animals were tested for each group. (D) Dietary Mg decreased the blood glucose levels as compared with DM mice during the i.p. glucose tolerance test. Five animals were tested for each group. One-way ANOVA with Bonferroni post hoc tests for multiple group comparisons; ${ }^{*} P<0.01$ vs. control, ${ }^{\#} P<0.05$ vs DM. CT, control mice; $\mathrm{CT}+\mathrm{Mg}$, control mice fed with normal chow and $\mathrm{Mg}$ in drinking water; DM, diabetic mice fed with high-fat diet; $\mathrm{DM}+\mathrm{Mg}$, mice fed with high-fat diet and $\mathrm{Mg}$ in drinking water.

$P<0.0001$ vs. $\mathrm{CT}$ and $P=0.21$ vs. $\mathrm{DM}+\mathrm{Mg}$ ). On the other hand, dietary $\mathrm{Mg}$ did not improve the decreased cytosol $\mathrm{Mg}$ level in DM mice ( $P=0.22$ vs. untreated DM mice), as shown in Figure 2B and Table 1. The change in plasma $\mathrm{Mg}$ had a modest effect on diabetic parameters. As shown in Figure $2 \mathrm{C}$ and Table 1, the fasting blood glucose in DM mice was decreased by dietary $\mathrm{Mg}$ from $270 \pm 17 \mathrm{mg} / \mathrm{dl}$ in DM to $216 \pm 7 \mathrm{mg} / \mathrm{dl}$ in DM $+\mathrm{Mg}(P<0.05)$. DM mice treated with $\mathrm{Mg}$ also showed an improvement in the glucose tolerance test (Figure 2D).

Dietary Mg supplementation improved cardiac DD and hypertrophy. Mg improved DD in DM mice (Figure 3). The incidence of DD decreased significantly from $90 \%$ of DM mice to $20 \% \mathrm{DM}+\mathrm{Mg}$ mice $(P$ $<0.0005)$. The $\mathrm{E} / \mathrm{e}^{\prime}$ returned to normal in $\mathrm{DM}+\mathrm{Mg}$ mice $(37 \pm 1$ in $\mathrm{DM}+\mathrm{Mg}$ vs. $45 \pm 2$ of $\mathrm{DM}, P<$ $0.0001)$. Figure $3 \mathrm{C}$ showed representative tissue Doppler echocardiography images of all 4 animal groups. $\mathrm{Mg}$ also reduced cardiac hypertrophy with decreased HW/TL ratio and PWTd, as shown in Figure 4. Mg did not alter the systolic or diastolic parameters of CT, suggesting that improvements in diastolic parameters were specific to $\mathrm{Mg}$-deficient mice. Moreover, in data not shown, we were able to demonstrate that $\mathrm{Mg}$ deficiency alone is sufficient to cause DD.

Dietary Mg supplementation improved mitochondrial function. Previously, we have reported that mitochondrial dysfunction can cause DD (20). Here, we investigated whether Mg improved cardiac DD through modulating mitochondrial function. As shown in Figure 5, DM resulted in a reduction in mitochondrial ATP synthesis ( $66 \pm 9 \mathrm{nmol} / 1$ per mg protein in DM vs. $89 \pm 6 \mathrm{nmol} / 1$ per mg protein in CT, $P<0.05$ ), increased mitochondrial $\mathrm{Ca}^{2+}$ load (3.71- \pm 1.28 -fold of $\left.\mathrm{CT}, P<0.0001\right)$, increased mitochondrial ROS (1.7- \pm 0.2 -fold of CT, $P<0.01$ ), and depolarized mitochondrial membrane potential $\left(\Delta \psi_{\mathrm{m}}\right)$ (JC-1 staining red to green fluorescence ratios; $0.65 \pm 0.06$ for DM vs. $1.72 \pm 0.18$ for $\mathrm{CT} ; P<0.01)$. Mg ameliorated all of these changes with increased ATP production (119 $\pm 10 \mathrm{nmol} / 1$ per mg protein, $P<0.01 \mathrm{vs}$. DM), decreased mitochondrial $\mathrm{Ca}^{2+}$ overload $(P<0.01$ vs. DM) and ROS overproduction $(P<0.05$ vs. DM), and 

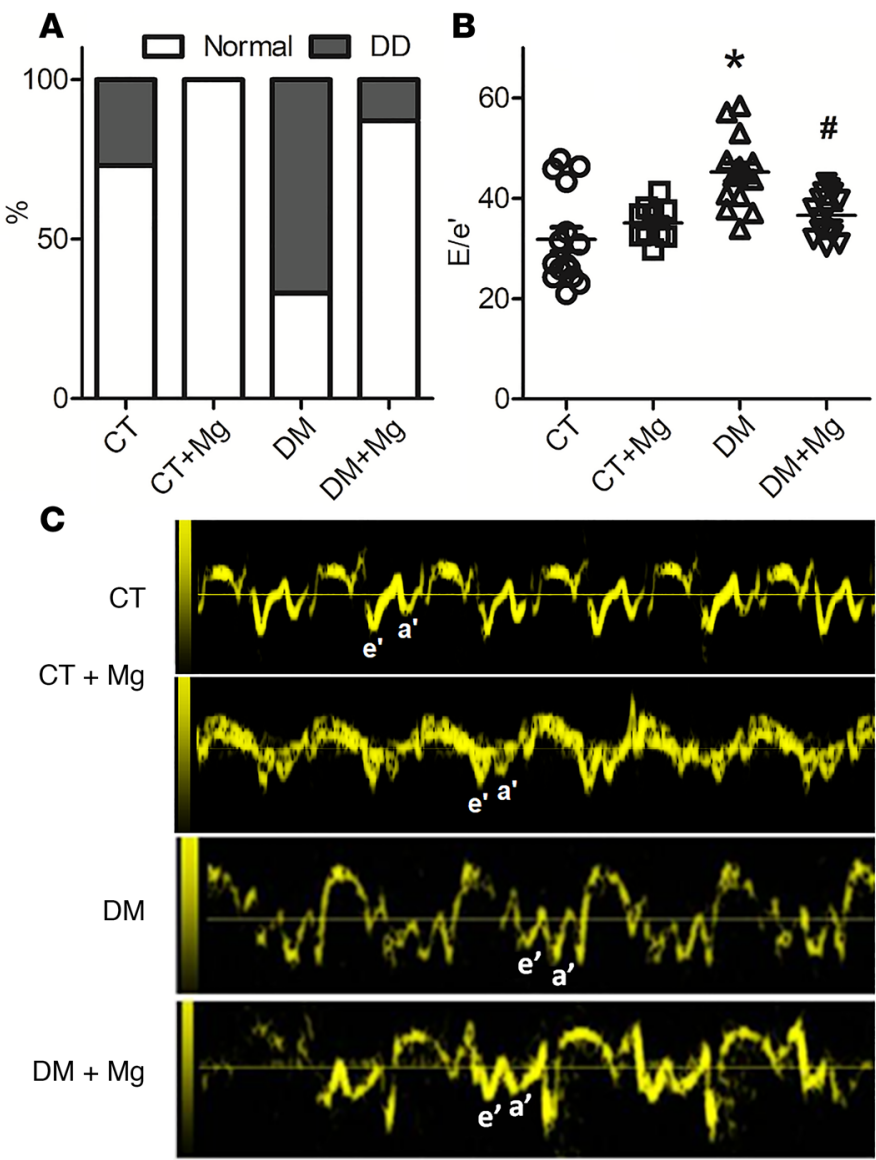

Figure 3. Dietary Mg improved cardiac diastolic dysfunction of DM mice. (A) Incidence of diastolic dysfunction (DD). (B) Mitral annulus TDI E/e'. (C) Representative tissue Doppler echocardiography images in control (CT), CT+Mg, DM, and $\mathrm{DM}+\mathrm{Mg}$ mice. Ten to 15 animals were tested for each group. One-way ANOVA with Bonferroni post hoc test and the $\chi^{2}$ Fisher exact test were used; ${ }^{*} P<0.0001$ vs. control and ${ }^{\#} P<0.001$ vs. DM. CT, control mice; $C T+M g$, control mice fed with normal chow and $\mathrm{Mg}$ in drinking water; $\mathrm{DM}$, diabetic mice fed with high-fat diet; $\mathrm{DM}+\mathrm{Mg}$, mice fed with high-fat diet and Mg in drinking water. DD, diastolic dysfunction; E/e', the ratio of transmitral Doppler early filling velocity to tissue Doppler early diastolic mitral annular velocity.

repolarized mitochondrial $\Delta \psi_{\mathrm{m}}(P<0.0001$ vs. DM). As we have shown before (20), mitochondrial morphology was disrupted by DM (Figure 6) with mitochondria swelling (indicated by increased mitochondrial size) and disrupted cristae structures (indicated by decreased gray intensity). Mg supplementation improved these changes. It seems likely that the Mg-mediated improvement in $\Delta \psi_{\mathrm{m}}$ and morphology, at least in part, account for the increase in ATP production with $\mathrm{Mg}$ supplementation.

$M g$-mediated changes in $M C U$. Figure 5 suggests that $\mathrm{Mg}$ supplementation alters mitochondrial $\mathrm{Ca}^{2+}$ handling. Therefore, we measured the levels of the principal mitochondrial $\mathrm{Ca}^{2+}$ entry channel, the mitochondrial $\mathrm{Ca}^{2+}$ uniporter (MCU). As shown in Figure 7, MCU was significantly increased in DM $(P<0.01$ vs. CT), and the changes were reversed by $\mathrm{Mg}$ (see complete unedited blots in the supplemental material; supplemental material available online with this article; https://doi.org/10.1172/jci. insight.123182DS1). The change in MCU represents one plausible molecular mechanism for the Mg-induced improvements in mitochondrial morphology and function.

\section{Discussion}

In this study, we found that HFD-induced DM was associated with hypomagnesemia, cardiac oxidative stress, disrupted mitochondrial function and morphology, cardiac hypertrophy, and DD. Mg supplementation improved all of these parameters (Figure 8).

Nearly $40 \%$ of heart failure patients present with low Mg levels at admission, and $72 \%$ have excessive urinary $\mathrm{Mg}$ loss (22). Low serum $\mathrm{Mg}$ predicts cardiovascular and all-cause mortality, as well as an increase 

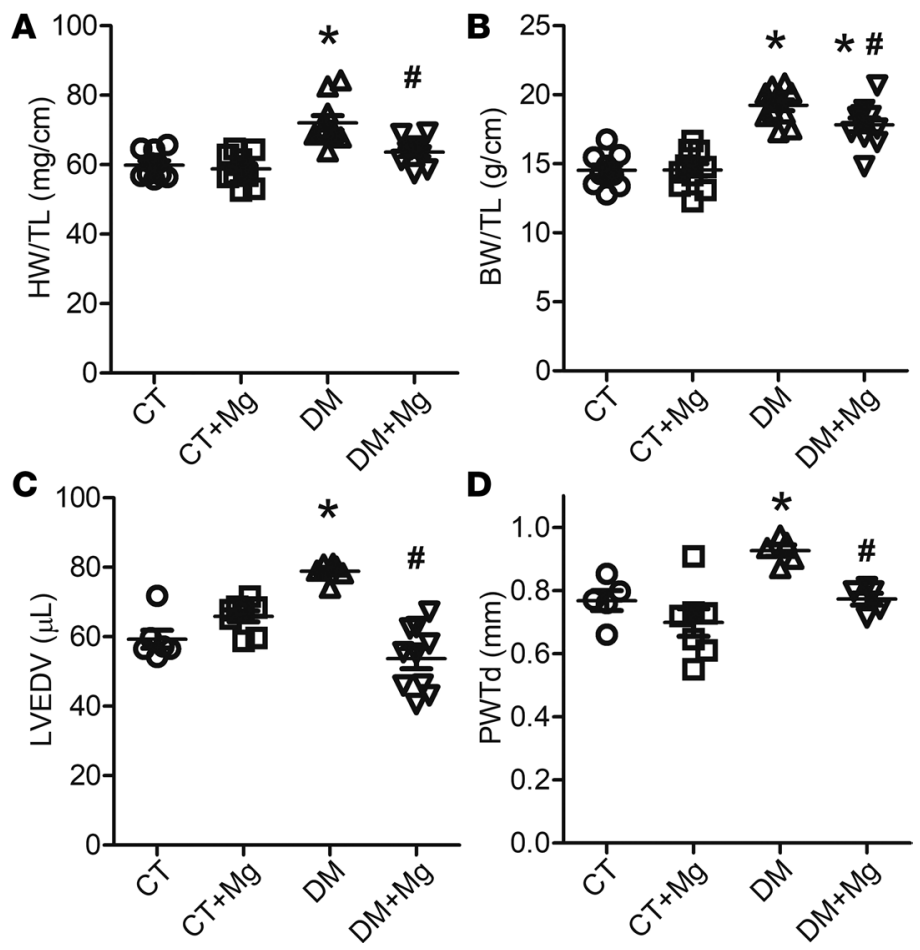

Figure 4. Dietary Mg mitigated cardiac hypertrophic changes in DM mice. (A) Heart weight/tibia length (HW/TL) Ten animals were tested for each group. (B) Body weight/tibia length (BW/TL). Ten animals were tested for each group. (C) Left ventricular end diastolic volume (LVEDV). Six to 10 animals were tested for each group. (D) LV diastolic posterior wall thickness (PWTd). Five to 7 animals were tested for each group. One-way ANOVA with Bonferroni post hoc test and 2-tailed Student's $t$ test were used; ${ }^{*} P<0.01 \mathrm{vs}$. control, ${ }^{\#} P<0.05$ vs. DM. CT, control mice; $\mathrm{CT}+\mathrm{Mg}$, control mice fed with normal chow and $\mathrm{Mg}$ in drinking water; $\mathrm{DM}$, mice fed with high-fat diet; $\mathrm{DM}+\mathrm{Mg}, \mathrm{mice}$ fed with high-fat diet and $\mathrm{Mg}$ in drinking water.

of left ventricular mass $(23,24)$. Several clinical trials have investigated the use of $\mathrm{Mg}$ in heart failure patients and have found that $\mathrm{Mg}$ supplementation improves heart rate variability (25) and arterial elasticity (26). When given as an adjuvant therapy in patients on optimal medical therapy, Mg improved heart failure survival and clinical symptoms (27). Nevertheless, the mechanism action of Mg remains unknown.

We have shown previously that mitochondrial dysfunction and the associated ROS overproduction can cause DD (20). In this study, Mg reversed DM-associated DD (Figure 3) and acted as a mitochondrial antioxidant (Figure 5C). This is likely to explain the effect of Mg on DD. Loss of Mg favors mitochondrial $\mathrm{Ca}^{2+}$ accumulation, shifting the equilibrium between $\mathrm{Ca}^{2+}$ transport and ATP synthesis toward the former reaction (28). One plausible mechanism for the $\mathrm{Mg}$ supplementation effect on mitochondria is a reversal of the DM-induced increase in the MCU (Figure 7). This idea is consistent with the observation that $\mathrm{Mg}$ perfusion of rabbit hearts after ischemia reduces mitochondrial $\mathrm{Ca}^{2+}$ overload and maintains mitochondrial ATP production (29). Furthermore, $\mathrm{Mg}$ can reverse the effects of $\mathrm{Ca}^{2+}$-induced $\Delta \psi_{\mathrm{m}}$ depolarization in isolated cardiac mitochondria (30) and inhibit mitochondrial ROS generation (31).

$\mathrm{Mg}$ may also work by improving glucose tolerance. In our study, Mg reduced fasting blood glucose and improved i.p. glucose tolerance in DM mice (Figure 2). Mg deficiency has been observed in human and animal DM (32-35). Hypomagnesemia (serum $\mathrm{Mg}<0.7 \mathrm{mmol} / \mathrm{l}$ ) accelerates type $2 \mathrm{DM}$ progression and increases the risk for diabetes complications. Moreover, $\mathrm{Mg}$ has been found to play a significant role in glucose and insulin metabolism, mainly through its impact on tyrosine kinase activity (36). Dietary Mg supplementation for patients with type $2 \mathrm{DM}$ improves glucose metabolism and insulin sensitivity (16). Nevertheless, our study showed that Mg presented significant protection against cardiac DD but only moderate effects on DM. Mg decreased the fasting blood glucose and enhanced the i.p. glucose tolerance, but it did not reverse the fasting serum insulin elevation in DM mice, suggesting that some of the effects of $\mathrm{Mg}$ on DD were independent of improved glucose control. It is unclear what percentage of the effect of $\mathrm{Mg}$ on DD was mediated by improvement in DM versus a direct effect on cardiac mitochondria. 

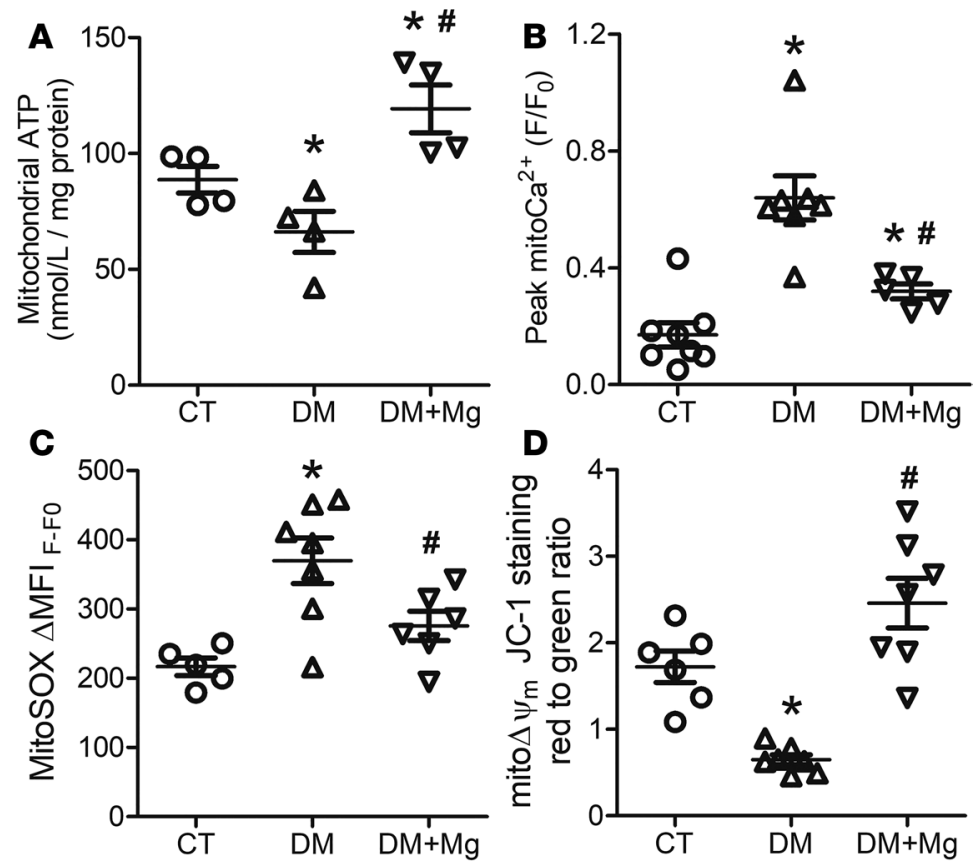

Figure 5. Dietary Mg suppressed mitochondrial oxidative stress and repolarized mitochondrial membrane potential in DM mice. (A) Mitochondrial ATP reduction in DM mice was improved by dietary Mg. Four animals were tested for each group. (B) Elevation of the peak mitochondrial $\mathrm{Ca}^{2+}$ transient in DM mice was reduced by dietary $\mathrm{Mg}$. Cardiomyocytes isolated from the left ventricle of 5-8 animals were tested for each group. (C) Overproduction of mitochondrial superoxide in DM mice was suppressed by dietary Mg. Cardiomyocytes isolated from the left ventricle of 5-7 animals were tested for each group. (D) Mitochondrial membrane potentials measured with JC-1 staining of ratios of red $(595 \mathrm{~nm})$ to green $(530 \mathrm{~nm})$ fluorescence were depolarized in DM mice and repolarized by dietary Mg. Cardiomyocytes isolated from the left ventricle of 6-7 animals were tested for each group. One-way ANOVA with Bonferroni post hoc test was used; ${ }^{*} P<0.01$ vs. control and ${ }^{*} P<0.05$ vs. DM. CT, control mice; DM, mice fed with high-fat diet; $\mathrm{DM}+\mathrm{Mg}$, mice fed with high-fat diet and $\mathrm{Mg}$ in drinking water. mito $\mathrm{Ca}^{2+}$, mitochondrial $\mathrm{Ca}^{2+} ; \Delta \mathrm{MFI}_{\mathrm{F}-\mathrm{FO}}$, The $\Delta$ mean of the fluorescence intensity obtained from the subtraction of background fluorescence $\left(\mathrm{F}-\mathrm{F}_{0}\right)$; mito $\Delta \psi_{\mathrm{m}}$, mitochondrial membrane potential.

In summary, DM induced mitochondrial dysfunction and caused cardiac DD. Mg improved the mitochondrial changes and reversed the DD. Therefore, dietary $\mathrm{Mg}$ supplementation may be an inexpensive therapy for DD and subsequent heart failure with preserved ejection fraction.

\section{Methods}

Diabetic mouse model. Male C57BL/6J mice were purchased from the Jackson Laboratory. Female mice did not develop DM (37) and were excluded. Mice were randomly assigned to 4 groups of 6- to 8-week old mice: (Group 1) CT fed with regular chow (Teklad Global, 18\% protein extruded rodent diet [sterilizable] from ENVIGO, catalog 2018SX) for 16 weeks; (Group 2) CT + Mg, CT mice fed with regular chow for 10 weeks and then given both regular chow and $\mathrm{MgSO}_{4}(50 \mathrm{mg} / \mathrm{ml}$ in the drinking water, approximately 6-8 g/kg/day) (21) for another 6 weeks; (Group 3) DM mice fed with HFD (60\% of calories from dietary fat (Research Diets, catalog \#D12492) for 16 weeks; and (Group 4) DM + Mg mice fed with HFD for 10 weeks and then given both $\mathrm{HFD}$ and $\mathrm{MgSO}_{4}(50 \mathrm{mg} / \mathrm{ml}$ in the drinking water, approximately $6-8 \mathrm{~g} / \mathrm{kg} /$ day) for another 6 weeks. Groups 1 and 3 were given water ad libitum. All tests were performed at 22-24 weeks of age.

Diagnosis of DM. Mice were fasted for approximately 7 hours. Fasting blood glucose and insulin levels were determined before glucose (1.5 g glucose $/ \mathrm{kg} \mathrm{BW}$ ) was administered by i.p. injection to determine glucose tolerance. Subsequently, the blood glucose levels were measured by a glucometer (ACCU-CHEK, Roche Applied Science) with blood drawn from the tail vein at 0, 15, 30, 90, and 120 minutes after the glucose injection. Fasting serum insulin levels were measured using an enzyme-linked immunosorbent assay kit (MilliporeSigma).

Noninvasive echocardiographic assessment. Noninvasive echocardiography was performed with a Vevo 2100 ultrasound system (VisualSonics), as we have done previously $(10,38,39)$. During the image 
A

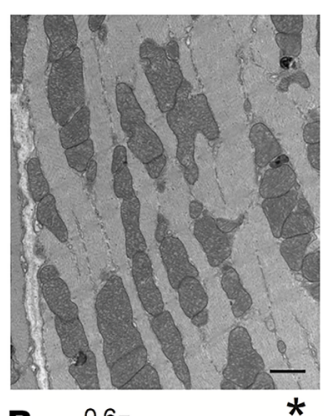

$\mathrm{DM}$
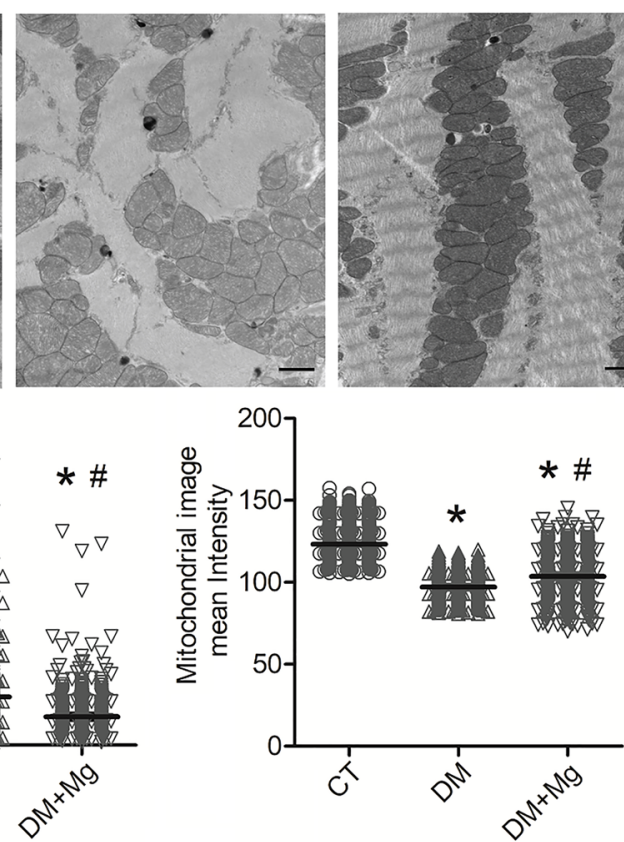

Figure 6. DM mice showed mitochondrial structural abnormalities, which were improved with dietary Mg. (A) Electron microscopy of mitochondria showed altered distribution and morphology in DM mouse heart tissue. These changes were improved by dietary Mg supplementation. Transmission electron micrographs of hearts from control mice (CT), DM mice, and DM+Mg mice. Images were taken at $\times 12,000$ magnification. Scale bars: $1 \mu \mathrm{m}$. (B) Mitochondria size was evaluated by the area of mitochondria, and the cristae structure was evaluated by the mitochondrial gray intensity mean values. Over 14 images for each group and 489-707 mitochondria were used for data analysis. One-way ANOVA with Bonferroni post hoc test was used; ${ }^{*} P<$ 0.01 vs. control and $\# P<0.05$ vs. DM. CT, control mice; DM, mice fed with high-fat diet; DM+Mg, mice fed with high-fat diet and Mg in drinking water.

acquisition, mice were anesthetized with $1 \%$ isoflurane, and a heart rate approximately 400 beats/ minute was maintained. M-mode images in the parasternal long axis and the LV short-axis views at the midpapillary level were taken. E/e' was assessed in the subcostal 4 chamber view by pulsed-wave and tissue Doppler imaging to evaluate the diastolic function (11). Measurements were averaged from 5 consecutive beats during expiration. The images for each mouse were recorded for at least 5 seconds (30-40 cardiac cycles). For the baseline evaluation, the following LV diastolic and systolic dimensions were measured: PWTd and PWT in systole, the anterior wall thickness in diastole and in systole, the LV end diastolic dimension (LVEDD), and LV end systolic dimension (LVESD). From these dimensions,

Figure 7. In DM mice, the protein level of MCU was elevated compared with control mice. Mg supplementation reversed the change. Heart tissues from 3 mice were used to collect protein samples and perform Western blot for each group. One-way ANOVA with Bonferroni post hoc test and 2-tailed Student's $t$ test were used; ${ }^{*} P<0.01$ vs. control and ${ }^{\#} P<0.05$ vs. DM. CT, control mice; $C T+M g$, control mice fed with normal diet and $\mathrm{Mg}$ in drinking water; DM, mice fed with high-fat diet; $\mathrm{DM}+\mathrm{Mg}$, mice fed with high-fat diet and $\mathrm{Mg}$ in drinking water. $\mathrm{MCU}$, mitochondrial $\mathrm{Ca}^{2+}$ uniporter.

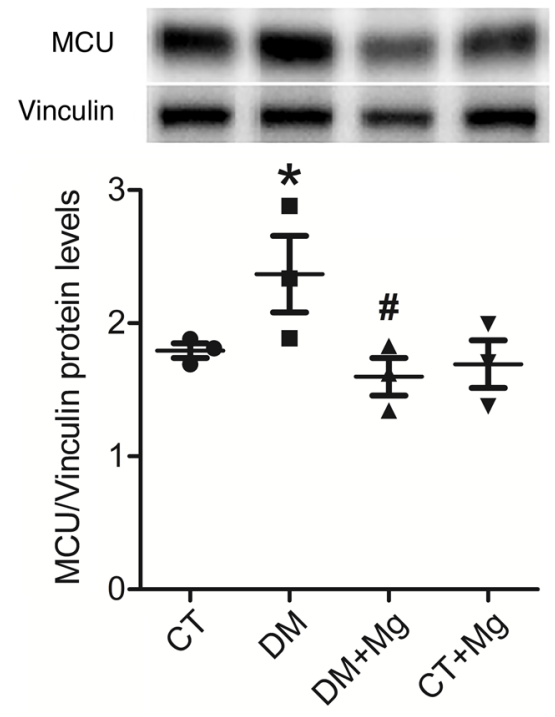




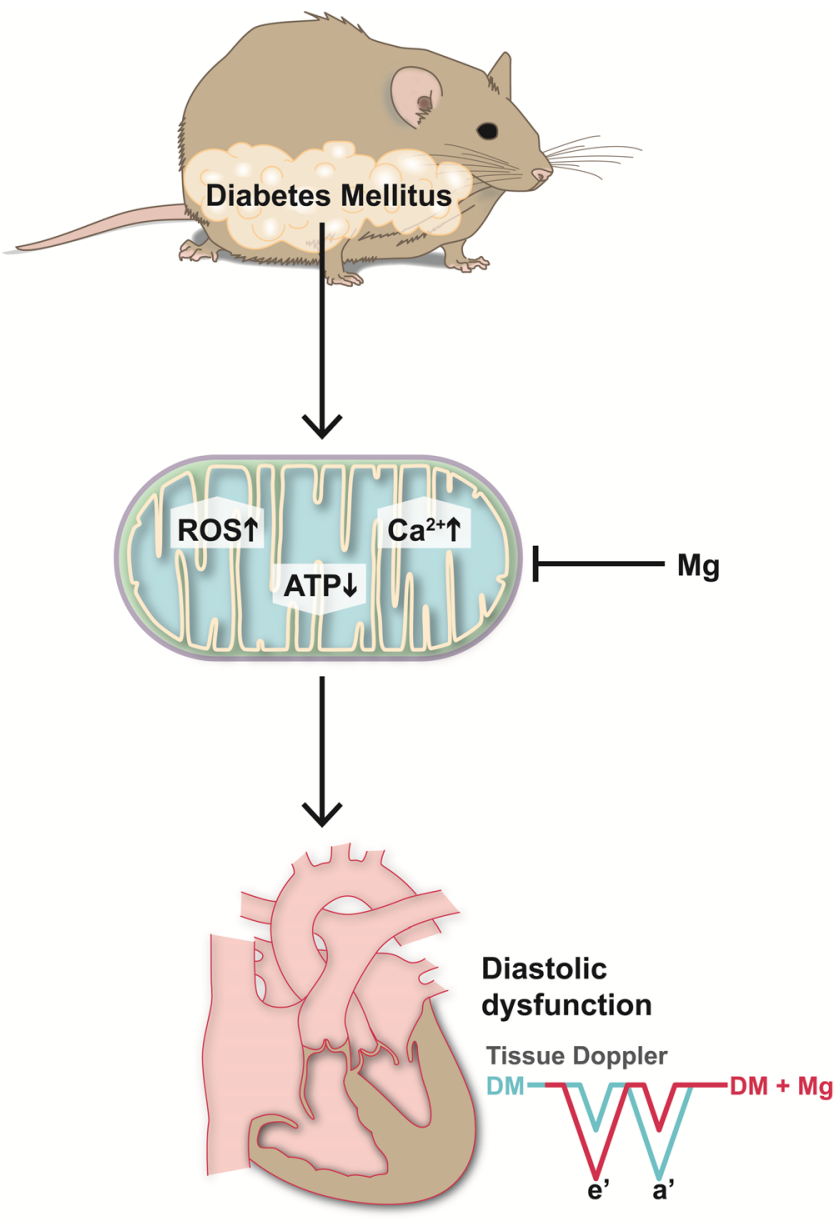

Figure 8. A scheme of high-fat diet-induced DM causing cardiac diastolic dysfunction by impairing mitochondria with increased mitochondrial ROS, decreased mitochondrial ATP production, and mitochondrial $\mathrm{Ca}^{2+}$ overload. Mg supplementation reverses these mitochondrial changes and improves diastolic function.

the main functional parameter - percent LV ejection fraction - was calculated as $100 \times(7 / 2.4+$ LVEDD) $\times \operatorname{LVEDD}^{3}-(7 / 2.4+\mathrm{LVESD}) \times \mathrm{LVESD}^{3} /(7 / 2.4+\mathrm{LVEDD}) \times \mathrm{LVEDD}^{3}$

Plasma Mg levels. Blood was collected in heparin-containing collection tubes and separated into plasma by centrifugation at $3,000 \mathrm{~g}$ for 20 minutes at $4^{\circ} \mathrm{C}$. Mg was determined with the QuantiChrom Magnesium Assay Kit (BioAssay System) by following the manufacturer's instructions. Briefly, plasma samples and dilutions of a standard Mg solution ( $0,0.2,0.4,0.6,0.8,1.0$, and $1.2 \mathrm{mmol} / \mathrm{l})$ were mixed with detecting reagents in microplates and incubated for 2 minutes at room temperature. The optical density (OD) was read at $500 \mathrm{~nm}$. Then, EDTA solution was added, and the OD was read again at $500 \mathrm{~nm}$ to obtain background absorbance. $\mathrm{Mg}$ concentration was calculated as $\left(\left[\mathrm{OD}_{\text {sample }}-\mathrm{OD}_{\text {blank }}\right] /\left[\mathrm{OD}_{\mathrm{Mg}}-\mathrm{OD}_{\text {Mgblank }}\right] \times 2[\mathrm{mg} / \mathrm{dL}]\right) \cdot \mathrm{OD}_{\text {sample }}$ and $\mathrm{OD}_{\text {blank }}$ were sample absorbance values before and after the addition of EDTA. $\mathrm{OD}_{\mathrm{Mg}}$ and $\mathrm{OD}_{\text {Mgblank }}$ were $\mathrm{OD}$ values of the standard before and after the addition of EDTA. Seven to 15 mice were tested for each group.

Isolation of ventricular cardiomyocytes. Adult mice ventricular cardiomyocytes were isolated as described before $(11,12)$. Briefly, hearts were excised under isoflurane $(2 \%)$ anesthesia and perfused with buffer (mmol/1: $113 \mathrm{NaCl}, 4.7 \mathrm{KCl}, 0.6 \mathrm{Na}_{2} \mathrm{HPO}_{4}, 0.6 \mathrm{KH}_{2} \mathrm{PO}_{4}, 1.2 \mathrm{MgSO}_{4}, 0.032$ phenol red, $12 \mathrm{NaH}-$ $\mathrm{CO}_{3}, 10 \mathrm{KHCO}_{3}, 10 \mathrm{HEPES}, 30$ taurine, 10 2-3-butanedione monoxime) for 7 minutes at $3 \mathrm{ml} / \mathrm{minute}$ flow rate. Hearts were then perfused with collagenase II $(0.8 \mathrm{mg} / \mathrm{ml}$, Worthington Biochemical Co.) for 10 minutes at $37^{\circ} \mathrm{C}$ using a temperature-controlled Langendorff perfusion system. The atria were removed. Following suspension with serially increasing $\mathrm{Ca}^{2+}$ concentrations $(0.2,0.5$, and $1 \mathrm{mmol} / \mathrm{l})$, cardiomyocytes were suspended immediately in a MEM containing $5 \% \mathrm{FBS}, 1 \%$ penicillin/streptomycin, $1 \mathrm{mmol} / 1$ pyruvate, $5.5 \mathrm{mM}$ glucose, and $1 \%$ insulin-transferrin-selenium (ITS) in a $95 \% / 5 \% \mathrm{O}_{2} /$ $\mathrm{CO}_{2}$ incubator at $37^{\circ} \mathrm{C}$. 
Cytosol Mg levels. The basal level of cytosol free $\mathrm{Mg}$ was measured using a specific Mg probe Mag-fluo-4 AM (10 $\mu \mathrm{mol} / 1$, Thermo Fisher Scientific) with fluorescence microscope (Olympus iX81). Isolated cardiomyocytes were loaded with Mag-fluo-4 AM for 40 minutes, followed by 30-minute deesterification of intracellular AM esters in a $95 \% / 5 \% \mathrm{O}_{2} / \mathrm{CO}_{2}$ incubator at $37^{\circ} \mathrm{C}$. Fluorescence signals $(\mathrm{F})$ and background $\left(\mathrm{F}_{0}\right)$ were recorded at excitation/emission of 488/520 nm with GFP filter at different extracellular $\mathrm{Mg}$ concentrations (0.5-2 mmol/1) as titration tests. The fluorescent signals $\left(\mathrm{F} / \mathrm{F}_{0}\right)$ were used to indicate cytosol $\mathrm{Mg}$ levels. Cytosolic $\mathrm{Mg}$ levels were obtained from 7-15 mice in each group. Eleven to 12 cardiomyocytes from each mouse were measured.

Mitochondrial ROS measurement. Mitochondrial ROS were measured by flow cytometry, as described before $(11,12)$. Briefly, isolated cardiomyocytes were incubated with MitoSOX Red $(5 \mu \mathrm{mol} / 1)$ (Thermo Fisher Scientific) for 15 minutes at $37^{\circ} \mathrm{C}$ and washed twice with modified standard Tyrode's solution (11). Ten thousand myocytes were assessed by flow cytometry (LSR, BD Biosciences). Cells were first gated with forward scatter by size and side scattered by garrulity. A second gating was performed by pulse-width to select single myocytes. MitoSOX Red was detected at excitation/emission of 510/580 $\mathrm{nm}$. Unstained myocytes were used as a reference standard $\left(\mathrm{F}_{0}\right)$. The $\Delta$ mean of the fluorescence intensity $(\triangle \mathrm{MFI})$ was obtained from the subtraction of background fluorescence $\left(\mathrm{F}-\mathrm{F}_{0}\right)$.

$\Delta \psi_{m}$ Measurement. The $\Delta \psi_{\mathrm{m}}$ was accessed by flow cytometry using a fluorescent probe, 5,5',6,6'-tetrachloro-1,1',3,3'-tetraethylbenzimidazolocarbo-cyanine iodide (JC-1, Invitrogen) by following the manufacturer's instructions. Briefly, isolated myocytes were washed with a MEM (containing in mmol/1: $1.8 \mathrm{Ca}^{2+}$, 5.5 glucose, and 5 pyruvate) and loaded with JC-1 (2 $\mu \mathrm{mol} / 1)$ for 20 minutes. Myocytes were then washed twice with the media and subjected to flow cytometry using 2 fluorescent channels: channel 1 red fluorescence and channel 2 green fluorescence. Ten thousand myocytes were gated as described above. When mitochondria are polarized electrically, JC-1 forms J-aggregates that emit orange-red fluorescence with a maximum at $595 \mathrm{~nm}$. J-monomers, indicating depolarized mitochondria, emit green fluorescence with a maximum at $530 \mathrm{~nm}$. A mitochondrial uncoupler, carbonilcyanide p-triflouromethoxyphenylhydrazone (FCCP), was added at the end of the sample recordings, and the readings were repeated. The $\Delta \psi_{\mathrm{m}}$ was calculated by a ratio of red/green fluorescence. A smaller ratio indicates mitochondria depolarization.

Mitochondrial ATP measurement. ATP was measured with EnzyLight ATP assay (BioAssay Systems) by following the manufacturer's instructions. Cells were incubated with or without oligomycin $(10 \mu \mathrm{mol} / 1)$ for 10 minutes. Cells were spun down at $12,000 \mathrm{~g}$ for 5 minutes and treated with $100 \mu$ of lysis buffer (in mmol/1: 50 Tris-HCl, $150 \mathrm{NaCl}, 5$ EDTA, 1\% Triton X-100 containing protease inhibitor cocktail; $\mathrm{pH}$ 7.4). Supernatant of cell lysates $(10 \mu 1)$ and ATP standards $(0-30 \mu \mathrm{mol} / 1)$ were mixed with $1 \mu \mathrm{l}$ of fresh D-luciferin reaction substrate, $1 \mu 1$ of ATP enzyme, and $90 \mu 1$ of reconstituted reagent. Luminescence was read immediately with a plate luminometer (model SMATBL, Synergymx, BioTek Instruments Inc.). Mitochondrial ATP was calculated by the subtraction of intracellular ATP level (with oligomycin) from total ATP (without oligomycin). Mitochondrial ATP was measured as nmol/1 per mg protein.

Measurement of mitochondrial $\mathrm{Ca}^{2+}$ transients. As we have done before $(40,41)$, mitochondrial $\mathrm{Ca}^{2+}$ transients were monitored by loading cardiomyocytes with Rhod-2 AM $\left(1 \mu \mathrm{mol} / 1,1\right.$ hour, $37^{\circ} \mathrm{C}$, Thermo Fisher Scientific) combined with the ruptured current-clamp technique with $20 \mu \mathrm{mol} / 1$ EGTA added in the pipette solution (in mmol/1: 120 potassium gluconate, $20 \mathrm{KCl}, 5 \mathrm{NaCl}, 5 \mathrm{HEPES}$, and $5 \mathrm{MgATP}$; $\mathrm{pH}$ 7.2). The extracellular bathing solution (Tyrode's solution) contained (in mmol/1) $140 \mathrm{NaCl}, 5.4 \mathrm{KCl}, 1 \mathrm{MgCl}_{2}, 10 \mathrm{HEPES}$, $1.8 \mathrm{CaCl}_{2}$, and 5.5 glucose ( $\mathrm{pH}$ 7.4). Because of its positive charge, Rhod-2 AM accumulates primarily in the mitochondrial matrix. Minor cytosolic traces of Rhod-2 were eliminated by whole-cell dialysis using the pipette solution $(42,43)$. Cardiomyocytes were paced at $1 \mathrm{~Hz}$ for 20 seconds. Rhod-2 intensity was sampled at a rate of $1 \mathrm{kHz}$ by an IonOptix system (IonOptix LLC). Mitochondrial $\mathrm{Ca}^{2+}$ transients were presented as background normalized fluorescence $\left[\left(\mathrm{F}-\mathrm{F}_{0}\right) / \mathrm{F}_{0}\right]$. Three consecutive traces were averaged for data analysis.

Electron microscopy. Cardiac mitochondrial morphology was observed by transmission electron microscopy (EM), as previously reported (20). Heart tissues were fixed with EM grade 4\% glutaraldehyde in 0.1 mol/1 cacodylate buffer. Fixed tissues were incubated with 1\% osmium tetroxide in the same buffer for 2 hours and processed for embedding. Ultra-thin sections were stained with uranyl acetate and lead citrate. All materials were purchased from Electron Microscopy Sciences. Samples were visualized with a JEM1220 Jeol transmission electron microscope, and micrographs were taken using a Gatan Digital Micrograph (Gatan Microscopy). Fifteen to 24 images were taken randomly from 3-5 mice in each group. To analyze the mitochondrial structure, we measured the mitochondrial area and gray mean intensity of each single mitochondrion in all images by ImageJ $(\mathrm{NIH})(44,45)$. 
Western blot of MCU. Heart tissue of 3 mice from each group were collected and processed for Western blotting. Anti-MCU was purchased from Cell Signaling Technology. Horseradish peroxidase-conjugated goat anti-rabbit IgG secondary antibodies (Bio-Rad, catalog 1706515) were used with dilution of 1:5000. Vinculin (Cell Signaling Technology) was used as a loading control.

Statistics. Data are presented as mean \pm SEM. For the dot plots, the lines indicated the mean values and the error bars indicated SEM values. The 2-tailed Student's $t$ test, 1-way ANOVA with Bonferroni post hoc tests for multiple group comparisons, and the $\chi^{2}$ Fisher exact test were used where appropriate. All statistical analyses were performed with GraphPad Prism 6.0. $P<0.05$ was considered statistically significant.

Study approval. Animal care and interventions were undertaken in accordance with the National Institute of Health (NIH) Guide for the Care and Use of Experimental Animals, and all animal protocols were approved by the Institutional Animal Care and Use Committees of the Lifespan or the University of Minnesota.

Chemicals and reagents were purchased from Sigma-Aldrich (St. Louis, MO), except as stated otherwise.

\section{Author contributions}

ML contributed to the manuscript including designing and conducting experiments, analyzing and interpreting data, drafting, revising and finalizing the manuscript. EMJ, HL, AX, and EYS conducted experiments, analyzed data, and drafted the manuscript. GS, GEJ, and AZ conducted experiments and analyzed data. SCD contributed to the manuscript including designing research studies, analyzing data, revising and finalizing the manuscript.

\section{Acknowledgments}

This work is supported by National Institutes of Health grant R01 HL106592 and HL104025 (to S.C. Dudley Jr.).

Address to correspondence: Samuel C. Dudley Jr., VCRC 286 - MMC 508, 425 Delaware Street, SE, Minneapolis, Minnesota 55455, USA. Phone:612.624.8970; Email: sdudley@umn.edu.

1. Jia G, DeMarco VG, Sowers JR. Insulin resistance and hyperinsulinaemia in diabetic cardiomyopathy. Nat Rev Endocrinol. 2016;12(3):144-153.

2. Caballero B. The global epidemic of obesity: an overview. Epidemiol Rev. 2007;29:1-5.

3. Bostick B, et al. Mineralocorticoid receptor blockade prevents Western diet-induced diastolic dysfunction in female mice. Am J Physiol Heart Circ Physiol. 2015;308(9):H1126-H1135.

4. From AM, Scott CG, Chen HH. The development of heart failure in patients with diabetes mellitus and pre-clinical diastolic dysfunction a population-based study. J Am Coll Cardiol. 2010;55(4):300-305.

5. Manrique $\mathrm{C}$, et al. Obesity and insulin resistance induce early development of diastolic dysfunction in young female mice fed a Western diet. Endocrinology. 2013;154(10):3632-3642.

6. Jia G, et al. Endothelial Mineralocorticoid Receptor Deletion Prevents Diet-Induced Cardiac Diastolic Dysfunction in Females. Hypertension. 2015;66(6):1159-1167.

7. Bostick B, et al. Daily exercise prevents diastolic dysfunction and oxidative stress in a female mouse model of western diet induced obesity by maintaining cardiac heme oxygenase-1 levels. Metab Clin Exp. 2017;66:14-22.

8. von Bibra H, St John Sutton M. Diastolic dysfunction in diabetes and the metabolic syndrome: promising potential for diagnosis and prognosis. Diabetologia. 2010;53(6):1033-1045

9. Owan TE, Hodge DO, Herges RM, Jacobsen SJ, Roger VL, Redfield MM. Trends in prevalence and outcome of heart failure with preserved ejection fraction. N Engl J Med. 2006;355(3):251-259.

10. Silberman GA, et al. Uncoupled cardiac nitric oxide synthase mediates diastolic dysfunction. Circulation. 2010;121(4):519-528

11. Jeong EM, et al. Tetrahydrobiopterin improves diastolic dysfunction by reversing changes in myofilament properties. $J$ Mol Cell Cardiol. 2013;56:44-54.

12. Liu M, et al. Mitochondrial dysfunction causing cardiac sodium channel downregulation in cardiomyopathy. J Mol Cell Cardiol. 2013;54:25-34.

13. Jeong EM, et al. Metabolic stress, reactive oxygen species, and arrhythmia. J Mol Cell Cardiol. 2012;52(2):454-463

14. Wester PO, Dyckner T. Magnesium and hypertension. J Am Coll Nutr. 1987;6(4):321-328.

15. Dyckner T, Wester PO. Potassium/magnesium depletion in patients with cardiovascular disease. Am J Med. 1987;82(3A):11-17.

16. Gommers LM, Hoenderop JG, Bindels RJ, de Baaij JH. Hypomagnesemia in Type 2 Diabetes: A Vicious Circle? Diabetes. 2016;65(1):3-13.

17. Nielsen FH, Milne DB, Klevay LM, Gallagher S, Johnson L. Dietary magnesium deficiency induces heart rhythm changes, impairs glucose tolerance, and decreases serum cholesterol in post menopausal women. J Am Coll Nutr. 2007;26(2):121-132.

18. Song Y, He K, Levitan EB, Manson JE, Liu S. Effects of oral magnesium supplementation on glycaemic control in Type 2 diabetes: a meta-analysis of randomized double-blind controlled trials. Diabet Med. 2006;23(10):1050-1056.

19. Dong JY, Xun P, He K, Qin LQ. Magnesium intake and risk of type 2 diabetes: meta-analysis of prospective cohort studies. Diabetes Care. 2011;34(9):2116-2122. 
20. Jeong EM, et al. Role of Mitochondrial Oxidative Stress in Glucose Tolerance, Insulin Resistance, and Cardiac Diastolic Dysfunction. J Am Heart Assoc. 2016;5(5):e003046.

21. Ravn HB, Korsholm TL, Falk E. Oral magnesium supplementation induces favorable antiatherogenic changes in ApoE-deficient mice. Arterioscler Thromb Vasc Biol. 2001;21(5):858-862.

22. Ceremuzyński L, Gebalska J, Wolk R, Makowska E. Hypomagnesemia in heart failure with ventricular arrhythmias. Beneficial effects of magnesium supplementation. J Intern Med. 2000;247(1):78-86

23. Reffelmann T, et al. Low serum magnesium concentrations predict cardiovascular and all-cause mortality. Atherosclerosis. 2011;219(1):280-284.

24. Reffelmann T, et al. Low serum magnesium concentrations predict increase in left ventricular mass over 5 years independently of common cardiovascular risk factors. Atherosclerosis. 2010;213(2):563-569.

25. Almoznino-Sarafian D, et al. Magnesium administration may improve heart rate variability in patients with heart failure. Nutr Metab Cardiovasc Dis. 2009;19(9):641-645.

26. Fuentes JC, Salmon AA, Silver MA. Acute and chronic oral magnesium supplementation: effects on endothelial function, exercise capacity, and quality of life in patients with symptomatic heart failure. Congest Heart Fail. 2006;12(1):9-13.

27. Stepura OB, Martynow AI. Magnesium orotate in severe congestive heart failure (MACH). Int J Cardiol. 2009;131(2):293-295.

28. Ferrari R, Williams A, Di Lisa F. The role of mitochondrial function in the ischaemic and reperfused myocarium. In: Caldarera C, Harris P, eds. Advances in Studies on Heart Metabolism. Bologna, Italy: Clueb Publications; 1982:245-255.

29. Ferrari R, et al. Myocardial recovery during post-ischaemic reperfusion: effects of nifedipine, calcium and magnesium. $J$ Mol Cell Cardiol. 1986;18(5):487-498.

30. Racay P. Effect of magnesium on calcium-induced depolarisation of mitochondrial transmembrane potential. Cell Biol Int. 2008;32(1):136-145

31. Kowaltowski AJ, Naia-da-Silva ES, Castilho RF, Vercesi AE. Ca2 ${ }^{+}$-stimulated mitochondrial reactive oxygen species generation and permeability transition are inhibited by dibucaine or Mg2+. Arch Biochem Biophys. 1998;359(1):77-81.

32. Gürlek A, Bayraktar M, Ozaltin N. Intracellular magnesium depletion relates to increased urinary magnesium loss in type I diabetes. Horm Metab Res. 1998;30(2):99-102.

33. Resnick LM, Altura BT, Gupta RK, Laragh JH, Alderman MH, Altura BM. Intracellular and extracellular magnesium depletion in type 2 (non-insulin-dependent) diabetes mellitus. Diabetologia. 1993;36(8):767-770.

34. Wallach S, Verch RL. Tissue magnesium content in diabetic rats. Magnesium. 1987;6(6):302-306.

35. Martins IJ. Magnesium deficiency and induction of NAFLD and type 3 diabetes in Australasia. Australasian Medical Journal. 2017;10(3):235-237.

36. Volpe SL. Magnesium in disease prevention and overall health. Adv Nutr. 2013;4(3):378S-383S

37. Pettersson US, Waldén TB, Carlsson PO, Jansson L, Phillipson M. Female mice are protected against high-fat diet induced metabolic syndrome and increase the regulatory T cell population in adipose tissue. PLoS ONE. 2012;7(9):e46057.

38. Xiao HD, et al. Mice with cardiac-restricted angiotensin-converting enzyme (ACE) have atrial enlargement, cardiac arrhythmia, and sudden death. Am J Pathol. 2004;165(3):1019-1032.

39. Simpson D, Liu H, Fan TH, Nerem R, Dudley SC. A tissue engineering approach to progenitor cell delivery results in significant cell engraftment and improved myocardial remodeling. Stem Cells. 2007;25(9):2350-2357.

40. Xie A, et al. Mitochondrial Ca2 ${ }^{+}$Influx Contributes to Arrhythmic Risk in Nonischemic Cardiomyopathy. J Am Heart Assoc. 2018;7(8):e007805.

41. Xie A, et al. Mitochondrial Ca2 ${ }^{+}$flux modulates spontaneous electrical activity in ventricular cardiomyocytes. PLoS ONE. 2018;13(7):e0200448.

42. Kohlhaas $\mathrm{M}$, et al. Elevated cytosolic $\mathrm{Na}^{+}$increases mitochondrial formation of reactive oxygen species in failing cardiac myocytes. Circulation. 2010;121(14):1606-1613

43. Maack C, Cortassa S, Aon MA, Ganesan AN, Liu T, O'Rourke B. Elevated cytosolic Na+ decreases mitochondrial Ca2+ uptake during excitation-contraction coupling and impairs energetic adaptation in cardiac myocytes. Circ Res. 2006;99(2):172-182.

44. Jhun BS, et al. Protein kinase D activation induces mitochondrial fragmentation and dysfunction in cardiomyocytes. $J$ Physiol (Lond). 2018;596(5):827-855.

45. O-Uchi J, et al. Overexpression of ryanodine receptor type 1 enhances mitochondrial fragmentation and Ca2 ${ }^{+}$-induced ATP production in cardiac H9c2 myoblasts. Am J Physiol Heart Circ Physiol. 2013;305(12):H1736-H1751. 